\title{
FRAGMENTAÇÃO MECÂNICA DE AMÊNDOAS DE CUPUAÇU (Theobroma grandiflorum) ${ }^{1}$
}

\author{
Fábio de Albuquerque VILALBA², Antonio MARSAIOLI JR. ${ }^{2 * *}$, Nelson Horacio PEZOA GARCIA ${ }^{3}$
}

\begin{abstract}
RESUMO
O cupuaçu (Theobroma grandiflorum) é uma fruta nativa da região amazônica brasileira cujas sementes convenientemente fermentadas, secas e torradas podem ser utilizadas para a obtenção de um produto similar ao chocolate. Este trabalho objetivou pesquisar uma forma alternativa de quebra da amêndoa seca sem torrar, através de um beneficiador de cilindros, seguida da utilização de um jogo de peneiras para a separação das frações fragmentadas. Os fatores estudados no rendimento da obtenção dos nibs (cotilédones fragmentados) e na verificação da pureza deste material (percentual de casca e gérmen) foram a umidade da amêndoa inteira e a velocidade diferencial dos cilindros de borracha do beneficiador. Pelos resultados obtidos, verificou-se que a umidade da amêndoa inteira foi a variável que apresentou maior influência na quebra. Os maiores percentuais de recuperação de nibs foram conseguidos na umidade de $5 \%$. A variação dos niveis da velocidade diferencial não causou diferença significativa no rendimento de obtenção dos nibs. Em relação aos valores dos percentuais de casca e gérmen nos nibs, nenhuma variável estudada apresentou influência significativa nos valores desta resposta, mesmo assim todos os resultados foram bastante satisfatórios, apresentando valores abaixo de $1,75 \%$. O rendimento deste processo alternativo está em torno de $90 \%$ de obtenção dos cotilédones encontrados em uma determinada massa de amêndoas. Como conclusão, o processo proposto constituiu-se em alternativa mecanizada tecnicamente válida, que apresentou bom rendimento de recuperação dos nibs, com percentuais de casca e gérmen abaixo do tolerado.

Palavras-chave: nibs; cotilédone; quebra; cupuaçu.
\end{abstract}

\section{SUMMARY}

MECHANICAL FRAGMENTATION OF CUPUASSU KERNELS (Theobroma grandiflorum). Cupuassu (Theobroma grandiflorum) is a native fruit from the Brazilian Amazon region whose seeds suitably fermented, dried and roasted can be used to manufacture a product similar to chocolate. This work aimed at devising an alternative way of cracking the dried but not roasted kernels by using a roller crusher, followed by using a set of sieves for separating the fractions. The variables studied for determining the nibs (scrapped cotyledons) acquisition yield as well the purity of such material (expressed as percent of shell and embryo fragments) were the moisture of the whole kernel and the differential speed of the double rollers of the crusher. The results confirmed that the moisture of the whole kernel was the variable that most influenced the cracking of kernels. The greater yield obtainment of nibs was reached at the $5 \%$ moisture level of the whole kernels. The variation of the double rollers differential speed did not present any significant difference as regards the nibs acquisition yield. As to the percent values of shell and embryo fragments, no studied variables have shown any significant difference on such a response values, even so the results were very well satisfactory, presenting values below the $1.75 \%$. The yield of this alternative process is $90 \%$ of the cotyledons acquisition from certain kernels mass. As a conclusion, the process proposed herewith set oneself up as a mechanized alternative, showing good nibs acquirement yield, and percent of shells and embryo below the tolerated value.

Keywords: nibs; cotyledon; cracking; cupuassu.

\section{1 - INTRODUÇÃO}

O cupuaçu é sem dúvida a fruta regional mais apreciada e consumida na Amazônia brasileira [9]. A fruta é tão conhecida na região Norte que se dispõe de inúmeros produtos derivados do cupuaçu, todos eles com sabores envoltos de grandes elogios. Seu consumo, antes restrito à região Norte, vem conquistando novos mercados no Brasil e no exterior, devido as suas características de sabor e aroma [6].

As sementes do cupuaçu representam em média $17 \%$ do peso da fruta. Os cotilédones destas sementes

\footnotetext{
Recebido para publicação em 10/06/2003. Aceito para publicação em 08/06/2004 (001146).

2. Universidade Estadual de Campinas (UNICAMP) - Faculdade de Engenharia de Alimentos - Departamento de Engenharia de Alimentos, Caixa Postal 6121, CEP: 13083-970, Campinas-SP, Brasil. E-mail: tonymars@fea.unicamp.br

3. Universidade Estadual de Campinas (UNICAMP) - Faculdade de Engenharia de Alimentos - Departamento de Tecnologia de Alimentos, Caixa Postal 6121, CEP: 13083-970, Campinas-SP, Brasil. E-mail: nelson@fea.unicamp.br

* A quem a correspondência deve ser enviada.
}

são um material bastante nobre e excelente para a confecção de um produto similar ao chocolate.

Até o momento, desconhece-se a existência de um estudo sobre o comportamento das amêndoas do cupuaçu durante a quebra, com a finalidade de separar a casca e os gérmens para a obtenção dos nibs. Um dos principais problemas é a resistência mecânica da casca das amêndoas do cupuaçu, pois ela é mais resistente e apresenta maior densidade em comparação à casca das amêndoas de cacau. No caso das amêndoas de cacau, a separação é feita através das diferenças de densidades do cotilédone e da casca, realizada em máquinas denominadas de "winnowing", que é uma combinação de peneiramento e elutriação a ar [8]; a casca das amêndoas de cacau é bem frágil, facilitando desta forma a sua fragmentação e separação dos nibs. Com certeza este é um dos principais fatores que ainda dificultam a obtenção do chocolate de cupuaçu em escala industrial, pois, na maioria das vezes as amêndoas não têm o seu valor reconhecido, tendo como resultado a sua transformação em adubo ou resíduo, mas atualmente, em algumas regiões do Norte do Brasil, estas sementes estão sendo exportadas para outros países como o Japão. 
Através de testes preliminares, envolvendo a quebra e separação das amêndoas de cupuaçu, foi verificado que uma forma alternativa de quebra das amêndoas utilizando um beneficiador de cilindros industrial, seguida da utilização de um jogo de peneiras para a separação, poderia ser uma boa metodologia para a obtenção dos nibs. Este trabalho foi realizado a partir desta metodologia de quebra e separação.

O trabalho tem como objetivo avaliar a utilização do beneficiador de cilindros na quebra das amêndoas de cupuaçu, fermentadas e secas, juntamente com a utilização do processo de separação por peneiras com o intuito de se obter a fração desejada nibs, sendo este, matéria-prima para a confecção de um produto análogo ao chocolate. O objetivo também inclui avaliação da influência dos fatores umidade e velocidade diferencial dos cilindros de borracha do beneficiador no rendimento e pureza dos nibs obtidos. O trabalho também tem o intuito de promover o desenvolvimento de uma tecnologia totalmente nacional, agregando valor a um componente do fruto cupuaçu, que no momento está sendo exportado em vez de ser promovido no Brasil.

Justifica-se a utilização de amêndoas fermentadas e secas, e não torradas, pelo fato de que o tempo de torração necessário para o desenvolvimento dos compostos precursores do chocolate, utilizando-se cotilédones na forma de nibs, é menor do que com as amêndoas inteiras, além de se obter um produto final com sabor superior.

\section{2 - MATERIAL E MÉTODOS}

As sementes de cupuaçu foram coletadas em maio de 2002 em Tomé-Açu (PA), época de final de safra na região. Estas sementes foram fornecidas pela Cooperativa Agrícola Mista de Tomé-Açu (CAMTA), que é um complexo agroindustrial nipo-brasileiro de fabricação de polpas de frutas congeladas. As sementes pertenciam ao cupuaçu da variedade Redondo.

Os processos de fermentação e secagem das sementes seguiram a metodologia proposta por VASCONCELOS [11], pela qual é utilizada uma caixa de madeira para a fermentação das sementes e na seqüência uma barcaça de secagem para a obtenção das amêndoas secas. Essas operações foram procedidas nas instalações da CAMTA. Foram fermentados e secos três lotes de $150 \mathrm{~kg}$ de sementes denominados de F1, F2 e F3 que correspondem, respectivamente, ao primeiro, segundo e terceiro lote fermentado. Terminado o período de fermentação de cada lote (sete dias), as amêndoas eram retiradas da caixa de madeira e levadas para a barcaça, onde eram espalhadas para serem secas uniformemente. O período de secagem durou em torno de dez dias até as amêndoas atingirem entre 5 6\% de umidade. Em seguida, as amêndoas eram embaladas em sacos duplos, de plástico e aniagem como reforço, sendo imediatamente despachadas para a FEA/UNICAMP, em Campinas, SP.

Na Figura 1 está esquematizado o fluxograma do processo adotado para a obtenção dos nibs. A etapa de quebra das amêndoas foi cumprida em beneficiador de cilindros industrial adaptado, de origem francesa, marca Gauthier, modelo DBR 800. As operações ilustradas no fluxograma definem quatro aberturas, entre o par de cilindros paralelos revestidos de borracha, de distância ajustável, usadas para a fragmentação das amêndoas, na seqüência de $7 \mathrm{~mm}, 5 \mathrm{~mm}, 2 \mathrm{~mm}$ e $0,4 \mathrm{~mm}$, ou seja, conforme ocorria a fragmentação das amêndoas, a distância entre os cilindros do beneficiador ia sendo diminuída. Além destes valores, também estão assinaladas as aberturas em milímetros das malhas das peneiras utilizadas logo após cada etapa de fragmentação das amêndoas.

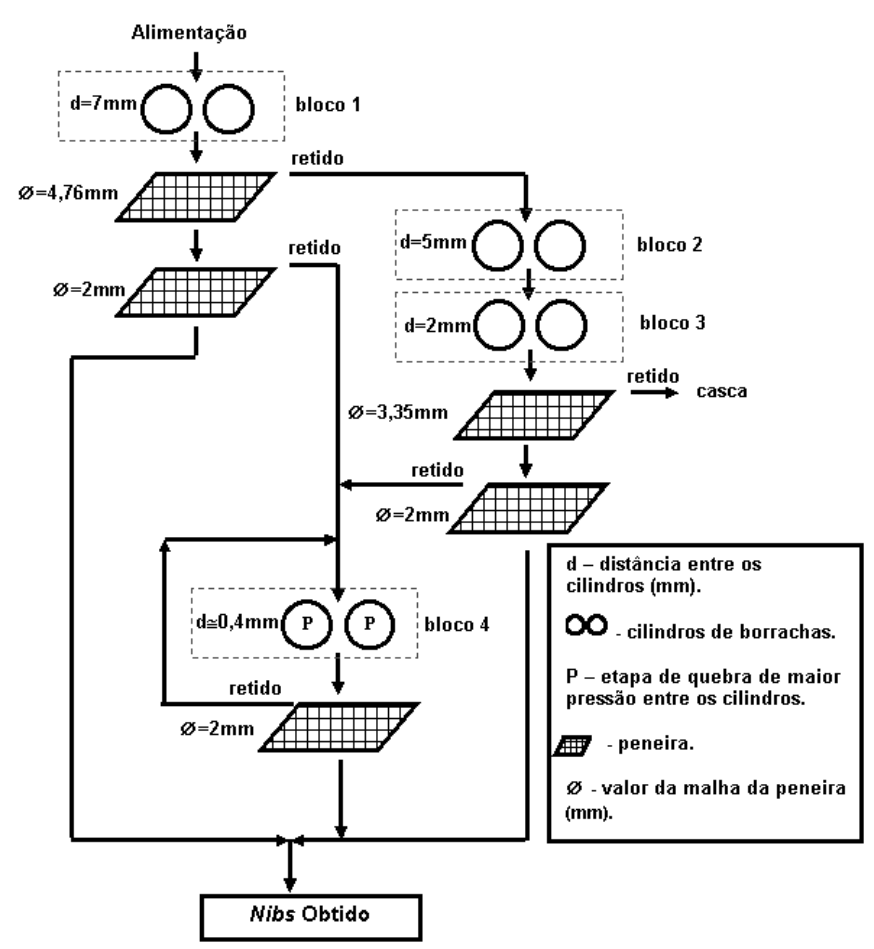

FIGURA 1. Fluxograma do processo de obtenção dos nibs.

Cada etapa de quebra foi denominada "bloco", correspondente a cada distância ajustada entre os cilindros de borracha. É visualizada na Figura 1 uma reciclagem do material a ser triturado no bloco 4 . Na primeira passagem do material o bloco é denominado $4 \mathrm{a}$, e na segunda passagem (reciclagem) bloco 4b. Um dos cilindros do equipamento está fixado em um par de mancais móveis, e através deste cilindro eram ajustadas as distâncias ao cilindro de borracha de mancais fixos. Ao cilindro móvel também estava vinculada uma mola de contra-pressão de carga ajustável, deixando este cilindro mais firme na sua posição pré-determinada ou, sob a condição de alimentação do beneficiador com algum material a ser triturado, permitindo pequenos movimentos do cilindro móvel, ajustando e acomodando melhor o material sob fragmentação. A carga da mola de contrapressão no cilindro móvel nos blocos 1,2 e 3 foi de $12,22 \mathrm{kgf}$, somente no final do processo (bloco 4) é que houve a necessidade da utilização de uma carga de maior valor $(56,40 \mathrm{kgf})$, pois as partículas que passam por este 
bloco são mais resistentes, de maior dificuldade de trituração para a obtenção dos nibs. A representação de um ajuste de carga maior na mola (bloco 4) é visualizada no fluxograma com a indicação da letra $P$ nos dois cilindros.

Para o processo de separação, foi utilizado um jogo de peneiras de aberturas 4,76, 3,35 e $2 \mathrm{~mm}$ (todas com dimensões $760 \times 740 \mathrm{~mm}$ ) para a separação das frações das amêndoas fragmentadas. O desempenho de quebra de cada bloco do fluxograma foi avaliado através do material que passou pela peneira de $2 \mathrm{~mm}$. Todo o material que não ficou retido nesta peneira compunha os nibs desejados, apresentando percentuais pequenos de casca e quase isenção de gérmen. O cálculo do desempenho de quebra de cada bloco é dado pela seguinte fórmula:

$\%=\frac{M_{C}}{M_{E}} \times 100$

$\%$ - Percentual de rendimento do bloco.

$M_{C}$ - Massa de material que passou pela peneira de 2mm (nibs obtidos).

$\mathrm{M}_{\mathrm{E}}$ - Massa de amêndoas fragmentadas no bloco.

Deve-se atentar para o fato de que, entre o bloco 2 e o bloco 3 , foi colocada uma peneira de $2 \mathrm{~mm}$, diferentemente do que é apresentado no fluxograma. Esta peneira apenas serviu para possibilitar um estudo de quais são os melhores ajustes dos fatores do bloco 2 . Em todas as etapas de peneiramento, sem exceção de nenhuma peneira, as amêndoas fragmentadas foram peneiradas durante 20 segundos, evitando assim erros nos valores das massas obtidas.

Também foram avaliados os percentuais de casca e gérmen encontrados nos nibs, estes obtidos em todas as etapas de quebra e separação dos ensaios de rendimentos. O trabalho foi realizado em triagem manual (separando-se nibs dos constituintes casca e gérmen) em amostras contendo aproximadamente 10g. Após a triagem manual, eram pesados os nibs dos outros constituintes que foram separados.

Utilizou-se para este trabalho dois fatores para o estudo da quebra das amêndoas: a velocidade diferencial entre os cilindros do beneficiador e a umidade da amêndoa inteira.

Começando pelo bloco 1 (Figura 1), foi utilizado um planejamento experimental $2^{2}$ com 4 pontos centrais. Em seguida, nos blocos 2, 3 e 4, fixou-se a umidade que apresentou melhor desempenho de quebra no bloco 1 e variou-se apenas a velocidade diferencial dos cilindros em cada bloco de quebra. Para avaliação dos blocos 2, 3 e 4 foi utilizado o teste de Tukey. Em todos os blocos de quebra foram avaliadas as seguintes respostas: rendimento dos nibs obtidos e o percentual de casca recolhido neste material. Para trabalhar com os resultados, foi utilizado o software STATISTICA 5.0 (executado em sistema operacional Windows ${ }^{\circledR}$ ) tanto para a análise dos efeitos do planejamento como para o teste de Tukey.

Por se tratar de um dos fatores que mais influenciaram a quebra, foi utilizada a umidade da amêndoa inteira como fator de estudo. Correspondendo a cada valor da umidade da amêndoa inteira, também foram obtidos os valores das umidades do cotilédone e da casca, os quais vão indicados na Tabela 1 .

TABELA 1. Valores das umidades das amêndoas de cupuaçu e seus componentes correspondentes a cada nível do planejamento experimental.

\begin{tabular}{cccc}
\hline \multirow{2}{*}{ Componentes } & \multicolumn{3}{c}{ Niveis } \\
\cline { 2 - 4 } & -1 & 0 & +1 \\
\hline Amêndoa inteira (\%) & $5,06 \pm 0,14$ & $5,93 \pm 0,38$ & $6,90 \pm 0,33$ \\
Casca (\%) & $10,07 \pm 0,29$ & $11,57 \pm 0,40$ & $13,03 \pm 0,93$ \\
Cotilédone (\%) & $3,41 \pm 0,25$ & $4,18 \pm 0,27$ & $5,02 \pm 0,41$ \\
\hline
\end{tabular}

Os níveis usados de umidade das amêndoas ficaram em torno de 5, 6 e 7\%, podendo-se justificar estes valores porque acima de $7 \%$ as amêndoas podem apresentar problemas de mofo $[6,7,8,11]$ e a obtenção de valores abaixo de $5 \%$ de umidade da amêndoa inteira é um processo difícil, somente com o processo de torração podem ser obtidas umidades menores. Para a determinação dos valores das umidades da amêndoa inteira, casca e cotilédones foi utilizada a metodologia 31.1.02 da AOAC [1].

Para elevar a umidade das amêndoas a $7 \%$ foi aplicada uma metodologia desenvolvida por AYDIN [2], onde ele estudou as propriedades físicas da noz de avelã em função da umidade. Dispondo-se de uma determinada massa de amêndoas de cupuaçu e de umidade inicial conhecida, pode ser calculado o percentual de água necessário para atingir a umidade desejada. Desta forma, as amêndoas foram borrifadas com a quantidade de água destilada necessária e seladas em sacos de polietileno. Estes sacos foram colocados em refrigerador com temperatura a $4^{\circ} \mathrm{C}$, permanecendo em torno de 8 dias até a distribuição, estabilização e uniformização da umidade por toda a amostra.

Para o ajuste de umidades menores, as amêndoas de cupuaçu eram colocadas em estufa de circulação de ar forçada da marca Fanem, modelo 330, por 24h, onde a temperatura não ultrapassava $35^{\circ} \mathrm{C}$. As amêndoas, ao atingirem a umidade desejada, eram submetidas aos processos de quebra e separação.

Para a quebra das amêndoas e separação na obtenção dos nibs foram utilizados os lotes F1 e F3. As amostras para a quebra foram preparadas previamente mediante a mistura de 50\% de F1 e 50\% de F3. Logo em seguida, estas misturas foram colocadas dentro de sacos de polietileno e permaneceram em refrigeradores a $5^{\circ} \mathrm{C}$ durante uma semana para que suas umidades entrassem em equilíbrio. Após uma semana, verificava-se a umidade dessa matéria-prima e, conforme o nível de umidade desejado para a análise da quebra, esta mistura ou era hidratada ou seca em estufa, conforme as metodologias apresentadas nos parágrafos anteriores.

Os valores de umidade das amêndoas inteiras, cascas e cotilédones puderam ser obtidos alternativamente de maneira mais rápida pelo Analisador de Umidade 
CEM, modelo Smart 5, que funciona a microondas. Para isto, o equipamento foi calibrado conforme a metodologia padrão apresentada acima.

Com relação ao fator velocidade diferencial dos cilindros, os sentidos de rotação de cada um são invertidos, denominando-se de velocidade diferencial do par de cilindros a diferença de valores das suas velocidades tangenciais.

Para escolher os niveis da velocidade diferencial no bloco 1, dentro do planejamento experimental, foi realizado um teste preliminar de quebra, resultando na escolha dos valores $2,10,2,66 \mathrm{e} 3,23 \mathrm{~m} / \mathrm{s}$. O intervalo de velocidades dos blocos 2 , 3 e 4 foi mais amplo, com velocidades de $0,84,1,87$ e $3,23 \mathrm{~m} / \mathrm{s}$, isto porque apenas o fator velocidade foi estudado para esses blocos.

\section{3 - RESULTADOS E DISCUSSÃO}

A matéria-prima ao chegar de Tomé-Açu (PA), foi caracterizada segundo a sua composição física, em termos dos percentuais de casca, cotilédone e gérmen, sendo seus valores apresentados na Tabela 2.

TABELA 2. Percentuais dos componentes das amêndoas de cupuaçu, fermentadas e secas, nos lotes F1, F2 e F3.

\begin{tabular}{cccc}
\hline \multirow{2}{*}{ Lotes } & \multicolumn{3}{c}{ Componentes } \\
\cline { 2 - 4 } & Testa (\%) & Gérmen (\%) & Cotilédone (\%) \\
\hline Lote F1 & $26,17 \pm 0,61$ & $0,64 \pm 0,03$ & $73,19 \pm 0,62$ \\
Lote F2 & $26,91 \pm 0,33$ & $0,53 \pm 0,08$ & $72,56 \pm 0,39$ \\
Lote F3 & $27,62 \pm 0,21$ & $0,69 \pm 0,04$ & $71,69 \pm 0,23$ \\
\hline
\end{tabular}

Valores médios em lotes de $100 \mathrm{~g}$ de amêndoas determinados em triplicatas.

O percentual de cotilédone encontrado em uma determinada massa de amêndoas de cupuaçu é importante pois, através deste dado, pode-se avaliar o rendimento do processo de obtenção dos nibs ao se passar as amêndoas pelas etapas de quebra e separação. Os valores apresentados na Tabela 2 estão de acordo com a literatura [6, 10].

Deve-se lembrar mais uma vez que, até o presente momento, não foi encontrado nenhum trabalho ou fontes bibliográficas sobre o estudo de quebra e obtenção dos nibs das amêndoas de cupuaçu, assim, os resultados que serão apresentados abaixo não terão nenhuma comparação.

A primeira etapa de quebra e separação, denominada de bloco 1 (Figura 1), foi estudada utilizando-se um planejamento experimental $2^{2}$ com quatro pontos centrais, sendo apresentados os resultados de percentuais dos nibs obtidos na Tabela 3. Observe-se que a carga da mola de pressão nesta primeira etapa de quebra, bloco 1 , foi de $12,22 \mathrm{kgf}$.

Na Tabela 4 são demonstrados os valores dos níveis das variáveis (velocidade diferencial e umidade) utilizadas no planejamento experimental.

A Tabela 5 apresenta a influência dos efeitos das variáveis (velocidade e umidade da amêndoa inteira) sobre as respostas dos percentuais dos nibs obtidos.
TABELA 3. Planejamento experimental $2^{2}$ com quatro pontos centrais (bloco 1)

\begin{tabular}{cccc}
\hline \multirow{2}{*}{ Ensaios } & \multicolumn{2}{c}{ Fatores } & Resposta \\
\cline { 2 - 4 } & $\begin{array}{c}\text { Velocidades } \\
\text { Diferenciais }\end{array}$ & Umidades & \% de nibs obtido \\
\hline A & -1 & -1 & 23,99 \\
B & +1 & -1 & 26,95 \\
C & -1 & +1 & 8,83 \\
D & +1 & +1 & 11,11 \\
E & 0 & 0 & 19,59 \\
F & 0 & 0 & 21,76 \\
G & 0 & 0 & 19,20 \\
H & 0 & 0 & 20,25 \\
\hline
\end{tabular}

TABELA 4. Valores dos níveis dos fatores utilizados na quebra das amêndoas no bloco 1 .

\begin{tabular}{cccc}
\hline Nível & -1 & 0 & +1 \\
\hline $\begin{array}{c}\text { Velocidade diferencial } \\
\text { entre os cilindros }(\mathrm{m} / \mathrm{s}) \\
\% \text { de umidade da } \\
\text { amêndoa inteira }\end{array}$ & 2,10 & 2,66 & 3,23 \\
\hline
\end{tabular}

TABELA 5. Análise dos efeitos sobre os percentuais de rendimentos.

\begin{tabular}{ccccccc}
\hline & Efeito & $\begin{array}{c}\text { Desvio } \\
\text { Padrão }\end{array}$ & $\mathrm{t}(3)$ & $\mathrm{p}$ & $\begin{array}{c}\text { Lim. Coef. } \\
-95 \%\end{array}$ & $\begin{array}{c}\text { Lim. Coef. } \\
+95 \%\end{array}$ \\
\hline Média* $^{*}$ & 18,960 & 0,398 & 47,598 & 0,0000 & 17,693 & 20,228 \\
(1) Velocidade & 2,619 & 1,127 & 2,324 & 0,1027 & $-0,967$ & 6,204 \\
(2) Umidade* & $-15,499$ & 1,127 & $-13,756$ & 0,0008 & $-19,084$ & $-11,913$ \\
1 X2 & $-0,342$ & 1,127 & $-0,303$ & 0,7816 & $-3,927$ & 3,244 \\
\hline
\end{tabular}

*Estatisticamente significativo $(\mathrm{p}<0,05)$. * Estatisticamente significativo $(\mathrm{p}<0,05)$.
Coeficiente de determinação $\mathrm{R}^{2}=0,912$, valido apenas para os parâmetros significa-
tivos (média e umidade).

Da Tabela 5 pode-se deduzir que a umidade é o fator que apresentou maior influência na resposta do planejamento: o sinal negativo do efeito da umidade indica que, conforme a umidade atinge niveis superiores, obtém-se menos nibs. No caso da velocidade, ao passar para niveis maiores a tendência é obter mais material, mas esta variável não apresenta um valor de $\mathrm{p}<0,05$ (confiabilidade de 95\%), o valor do seu efeito é baixo, assim, a variável velocidade não é estatisticamente significativa para esta confiabilidade. Nota-se a existência de valores pequenos no desvio padrão, isto significa que as repetições dos pontos centrais foram satisfatórias. O valor do coeficiente de determinação também está bastante satisfatório. Através destes resultados foram obtidos dois modelos matemáticos codificados fornecidos pelo planejamento experimental. O primeiro modelo é demonstrado pela equação 2 e apresenta 95\% de confiabilidade $(\mathrm{p}<0,05)$. Já o modelo com $89 \%$ de confiabilidade ( $<<0,11$ ) é visualizado na equação 3 e apresenta, como diferença em relação ao outro modelo, a inclusão do parâmetro velocidade. Tanto o modelo da equação 2 como o da equação 3 são codificados, isto é, os valores dos fatores devem ser aplicados nos 
modelos em $+1,0$ ou -1 . Deve-se lembrar que antes de começar a trabalhar com modelagem matemática, há necessidade de conferir a ANOVA (Analysis of Variance) do modelo gerado pelo planejamento experimental, servindo para verificar a qualidade do ajuste do modelo, isto é, se ele apresenta ou não muitos resíduos, neste caso, é chamado de resíduo a diferença entre um valor observado e a sua estimativa de acordo com o modelo. Tanto os modelos de 95\% quanto os de $89 \%$ de confiabilidade, apresentaram pela ANOVA resultados bastante satisfatórios, confirmando a validade de ambos os modelos e permitindo que sejam utilizados.

$\% \mathrm{C}=18,96-7,749 \mathrm{U}$

Coeficiente de determinação $R^{2}=0,912$, válido apenas para os parâmetros significativos (média e umidade)

$\% \mathrm{C}=18,96+1,309 \mathrm{~V}-7,749 \mathrm{U}$

Coeficiente de determinação $R^{2}=0,938$, válido apenas para os parâmetros significativos (média, velocidade e umidade).

Sendo:

$\% \mathrm{C}$ - Percentual de nibs obtido.

$\mathrm{V}$ - Velocidade diferencial entre os cilindros.

U - Umidade da amêndoa inteira.

Através dos modelos matemáticos codificados fornecidos pelas equações 2 e 3 é possivel avaliar o ajuste dos valores determinados pelos modelos (valores preditos) com os valores reais (observados) fornecidos através dos experimentos. Na Tabela 6 são visualizados os valores observados juntamente com os valores preditos pelos modelos propostos com 95\% e 89\% de confiabilidade, facilitando assim a análise de qual destes modelos matemáticos se ajusta melhor aos valores observados experimentalmente.

TABELA 6. Percentual de diferença entre os valores observados e os valores preditos.

\begin{tabular}{cccccccc}
\hline & \multicolumn{3}{c}{ Fatores } & $\begin{array}{c}\text { Respostas } \\
\text { Observadas }\end{array}$ & \multicolumn{2}{c}{ Modelos Codificados e Erros } \\
\hline Ensaios & Velocidade & Umidade & $\begin{array}{c}\text { \% nibs. } \\
\text { Obt. }\end{array}$ & $95 \%$ Conf. & $\begin{array}{c}\text { Erro } \\
(95 \%)\end{array}$ & $\begin{array}{c}89 \% \\
\text { Conf. }\end{array}$ & $\begin{array}{c}\text { Erro } \\
(89 \%)\end{array}$ \\
\hline A & -1 & -1 & 23,99 & 26,71 & 11,34 & 25,4 & 5,88 \\
B & 1 & -1 & 26,95 & 26,71 & 0,89 & 28,02 & 3,97 \\
C & -1 & 1 & 8,83 & 11,21 & 26,91 & 9,90 & 12,08 \\
D & 1 & 1 & 11,11 & 11,21 & 0,90 & 12,52 & 12,69 \\
E & 0 & 0 & 19,59 & 18,96 & 3,22 & 18,96 & 3,22 \\
F & 0 & 0 & 21,76 & 18,96 & 12,87 & 18,96 & 12,87 \\
G & 0 & 0 & 19,20 & 18,96 & 1,25 & 18,96 & 1,25 \\
H & 0 & 0 & 20,25 & 18,96 & 6,37 & 18,96 & 6,37 \\
\hline \multicolumn{7}{l}{ Média dos valores dos erros } \\
\hline
\end{tabular}

A fórmula do erro é fornecida por LOMAURO, BAKSHI \& LABUZA [5], onde é apresentada da seguinte maneira:

$$
\text { Erro }=\frac{\left|V_{o}-V_{p}\right|}{V_{o}} x 100
$$

$\mathrm{V}_{\mathrm{o}}=$ Valores observados experimentalmente.

$\mathrm{V}_{\mathrm{p}}=$ Valores preditos pelo modelo proposto.

Pela Tabela 6, o modelo com 89\% de confiabilidade apresenta média nos valores dos erros $(7,29 \%)$ inferior ao do modelo proposto com $95 \%(7,97 \%)$. Não se pode também ignorar que alguns valores dos erros aumentaram no modelo de $89 \%$ em relação ao modelo de $95 \%$ (ensaios B e D), só que estes valores são inferiores ou próximos de $10 \%$, que é mais ou menos o valor limite de erro aceitável. Um dado também a favor do modelo codificado com $89 \%$ de confiabilidade é que este apresentou coeficiente de determinação de $\mathrm{R}^{2}=0,938$, superior ao modelo de $95 \%\left(\mathrm{R}^{2}=0,912\right)$. A inclusão do termo velocidade é vantajosa, pois, além de diminuir a média dos valores dos erros, é uma variável fácil de ser ajustada, ao contrário da umidade da amêndoa.

Em termos de praticidade, transformando o modelo matemático codificado da equação $3 \mathrm{em}$ um modelo para valores reais com $89 \%$ de confiabilidade (velocidade em $\mathrm{m} / \mathrm{s}$ e umidade em percentual), obtém-se a seguinte equação:

$\% \mathrm{C}=63,36+2,30 \mathrm{Vr}-8,48 \mathrm{Ur}$

Coeficiente de determinação $\mathrm{R}^{2}=0,952$, válido apenas para os parâmetros significativos (média, velocidade e umidade).

Sendo:

$\% \mathrm{C}$ - Percentual de nibs obtidos.

Vr - Velocidade diferencial entre os cilindros $(\mathrm{m} / \mathrm{s})$.

Ur - Umidade da amêndoa inteira (\%).

O modelo empírico da equação 5 é linear local, isto é, ele é aplicável apenas a uma determinada região [3]. A inclusão de valores extrapolados no modelo (umidades maiores que $7 \%$ e menores que $5 \%$ e velocidades menores que $2,10 \mathrm{~m} / \mathrm{s}$ e maiores que $3,23 \mathrm{~m} / \mathrm{s}$ ) não é recomendada, pois o modelo foi estudado dentro de uma faixa pré-estabelecida.

No caso dos blocos 2, 3 e 4, foi utilizado o teste de Tukey para avaliação do desempenho dos rendimentos dos nibs obtidos, através das mudanças dos níveis das velocidades diferenciais entre os cilindros. A umidade das amêndoas inteiras foi fixada em torno de $5 \%$, pois foi a que apresentou os melhores resultados no descasque no bloco 1 . A carga da mola nos blocos 2 e 3 foi também fixada em 12,22kgf, somente no bloco 4 é que a carga foi aumentada em torno de 56,40kgf devido à partícula aí presente nessa etapa ser mais resistente à trituração.

Os resultados da Tabela 7 denotam que a mudança da velocidade diferencial entre os cilindros não implica em diferença significativa no rendimento da obtenção dos nibs (95\%), somente no bloco 4a houve uma diferença no rendimento entre a maior e a menor velocidade.

Quanto aos percentuais de casca e gérmen contidas nos cotilédones obtidos nos testes de quebra e rendimento, os resultados são apresentados nas Tabelas 8 e 10, fornecendo a pureza deste material. 
TABELA 7. Desempenho de quebra para diferentes velocidades diferenciais em cada bloco.

\begin{tabular}{ccccc}
\hline \multirow{2}{*}{$\begin{array}{c}\text { Velocidade diferencial } \\
\text { entre os cilindros }(\mathrm{m} / \mathrm{s})\end{array}$} & \multicolumn{4}{c}{ Percentual médio de nibs obtido em cada bloco } \\
\cline { 2 - 5 } & Bloco 2 & Bloco 3 & ${\text { Bloco } 4 \mathrm{a}^{\star}}$ & Bloco 4b** \\
\hline 0,84 & $13,5 \pm 0,88^{\mathrm{A}}$ & $34,49 \pm 0,65^{\mathrm{A}}$ & $62,31 \pm 2,49^{\mathrm{A}}$ & $36,47 \pm 1,50^{\mathrm{A}}$ \\
1,87 & $15,10 \pm 0,40^{\mathrm{A}}$ & $34,25 \pm 3,045^{\mathrm{A}}$ & $64,99 \pm 1,70^{\mathrm{AB}}$ & $35,83 \pm 3,28^{\mathrm{A}}$ \\
3,23 & $15,19 \pm 0,81^{\mathrm{A}}$ & $36,85 \pm 1,76^{\mathrm{A}}$ & $67,37 \pm 1,50^{\mathrm{B}}$ & $35,04 \pm 4,61^{\mathrm{A}}$ \\
\hline
\end{tabular}

Médias com expoentes iguais por bloco, na mesma coluna, não apresentam diferen-

cas significativas $(\mathrm{p}<0,05)$.
Todos os valores obtidos em triplicatas; *Primeira passagem no bloco 4 ; **Segunda passagem no bloco 4 .

Em relatório apresentado na reunião da comissão do CODEX ALIMENTARIUS [4], foi convencionado que o liquor do cacau não pode ultrapassar $1,75 \%$ de casca em sua massa. Como o derivado do cupuaçu, similar ao chocolate, ainda não é um produto comercializado em escala mundial, não há legislação, normas ou padrões para o mesmo. Devido a este fato, os padrões seguidos neste trabalho baseiam-se nos dos produtos do cacau e chocolate estabelecidos pelos comitês internacionais. MINIFIE [8] também cita que o nibs do cacau comercializado pode conter em torno de $1,5 \%$ de casca e gérmen.

Tabela 8. Planejamento experimental $2^{2}$ com quatro pontos centrais para análise do percentual de casca nos nibs no bloco 1.

\begin{tabular}{cccc}
\hline \multirow{2}{*}{ Ensaios } & \multicolumn{2}{c}{ Fatores } & Resposta \\
\cline { 2 - 4 } & Velocidades & Umidades & \% de casca no nibs \\
\hline A & -1 & -1 & 0,4030 \\
B & +1 & -1 & 0,3459 \\
C & -1 & +1 & 0,6026 \\
D & +1 & +1 & 0,3447 \\
E & 0 & 0 & 0,3756 \\
F & 0 & 0 & 0,2385 \\
G & 0 & 0 & 0,3532 \\
H & 0 & 0 & 0,2960 \\
\hline
\end{tabular}

Os valores dos niveis de cada fator (velocidade diferencial e umidade) são os mesmos da Tabela 4.

TABELA 9. Análise dos efeitos sobre os percentuais de cascas nos nibs.

\begin{tabular}{ccccccc}
\hline & Efeito & $\begin{array}{c}\text { Desvio } \\
\text { Padrão }\end{array}$ & $\mathrm{t}(3)$ & $\mathrm{p}$ & $\begin{array}{c}\text { Lim. Coef. } \\
-89 \%\end{array}$ & $\begin{array}{c}\text { Lim. Coef. } \\
+89 \%\end{array}$ \\
\hline Média* $^{*}$ & 0,370 & 0,022 & 17,017 & 0,000 & 0,321 & 0,419 \\
(1) Velocidade & $-0,158$ & 0,061 & $-2,561$ & 0,083 & $-0,296$ & $-0,019$ \\
(2) Umidade & 0,099 & 0,061 & 1,613 & 0,205 & $-0,039$ & 0,238 \\
1 X2 & $-0,100$ & 0,061 & $-1,633$ & 0,201 & $-0,239$ & 0,038 \\
\hline
\end{tabular}

*Estatisticamente significativo $(\mathrm{p}<0,11)$

Coeficiente de determinação $\mathrm{R}^{2}=0,312$; válido apenas para os parâmetros siónificativos (média e umidade).

Nota-se pela Tabela 9 que, para um valor de confiabilidade a $89 \%(\mathrm{p}<0,11)$, nenhum parâmetro é estatisticamente significativo com exceção da média e velocidade. O que se pode concluir é que, conforme é aumentado o valor da umidade da amêndoa, o nível de percentual de casca também se eleva, sendo que para a velocidade ocorre o inverso (fatos observados no bloco 1). O baixo valor do coeficiente de determinação $\left(\mathrm{R}^{2}=0,312\right)$ mostra que o modelo está muito mal ajustado em relação aos valores observados experimentalmente. Para este caso, para melhorar o valor do coeficiente de determinação, deve-se transformar esse modelo linear em quadrático. Para isto são necessários inserir pontos axiais no planejamento, isto é, novos níveis com pontos abaixo de -1 e acima de +1 para os fatores umidade e velocidade. No caso de um planejamento fatorial $2^{2}$, os valores dos pontos axiais seriam $-1,41 \mathrm{e}+1,41$. Só que, como já foi observado, não é possivel trabalhar com valores menores e maiores de umidades das amêndoas, e o processo de obtenção dos nibs foi estudado em uma faixa limite. Umidades maiores acarretariam o perigo das amêndoas mofarem ou de surgir bolor, e umidade menores exigiria temperaturas maiores na estufa de secagem, podendo iniciar um processo de torração nas amêndoas $[6,7,8,11]$.

Na Tabela 10, nota-se que, com exceção do bloco 3 , conforme a velocidade do equipamento é aumentada, menor é o percentual de cascas no material coletado.

TABELA 10. Percentual de casca encontrado nos blocos 2 , 3 e 4 .

\begin{tabular}{ccccc}
\hline \multirow{2}{*}{$\begin{array}{c}\text { Velocidade diferencial } \\
\text { entre os cilindros }(\mathrm{m} / \mathrm{s})\end{array}$} & \multicolumn{4}{c}{ Percentual médio de cascas em cada bloco } \\
\cline { 2 - 5 } & Bloco 2 & Bloco 3 & Bloco 4a $^{*}$ & Bloco 4b \\
\hline 0,84 & $2,801 \pm 0,20^{\mathrm{A}}$ & $1,360 \pm 0,25^{\mathrm{A}}$ & $1,402 \pm 0,46^{\mathrm{A}}$ & $2,660 \pm 0,77^{\mathrm{A}}$ \\
1,87 & $2,661 \pm 0,30^{\mathrm{A}}$ & $1,656 \pm 0,46^{\mathrm{AB}}$ & $1,024 \pm 0,28^{\mathrm{AB}}$ & $2,386 \pm 0,26^{\mathrm{A}}$ \\
3,23 & $2,125 \pm 0,43^{\mathrm{A}}$ & $2,212 \pm 0,38^{\mathrm{B}}$ & $0,948 \pm 0,17^{\mathrm{B}}$ & $2,378 \pm 0,38^{\mathrm{A}}$
\end{tabular}

As médias com expoentes iguais em cada bloco, na mesma coluna, não apresentam diferenças significativas $(\mathrm{p}<0,05)$; *Primeira passagem no bloco 4 ; **Segunda passagem no bloco 4 .

Outro detalhe importante, o material que alimentou os testes do bloco 3 , foi o mesmo que passou pelo bloco 2 na velocidade $3,23 \mathrm{~m} / \mathrm{s}$.

Vale observar que toda a matéria-prima obtida nesta primeira etapa de quebra (bloco 1) apresenta valores percentuais excelentes de casca e gérmen. O valor máximo de casca encontrado nos nibs foi $0,6026 \%$ ( $T a-$ bela 8). A Tabela 10 mostra que os valores apresentados nos blocos 2, 3, 4 são elevados, acima do permitido [5,9]. Com o intuito de esclarecer, estes valores de pureza são de pontos isolados, o valor mais importante é o da pureza do material obtido no final do fluxograma de quebra da Figura 1.

Ao se examinar a Tabela 10, o teste de Tukey demonstra que não houve diferença significativa, entre os valores dos blocos 2 e 4b, no percentual de casca e gérmen na mudança da velocidade diferencial dos cilindros. Todos os valores das Tabelas 3, 7, 8 e 10 (percentual dos nibs obtidos em todos os blocos e os percentuais de casca de todos estes nibs) e mais os valores das massas (que também foram pesadas durante os experimentos) das amêndoas fragmentadas que ficavam retidas em cada etapa de peneiramento, são mostrados na Tabela 11 .

Através desta tabela, que é uma matriz completa do comportamento de quebra do descascador de rolos, é possivel perceber o direcionamento das massas de 
cada granulometria, o percentual de rendimento dos nibs de cada bloco, e o percentual de casca do material recolhido, entre outros dados.

Para avaliação do modelo será feita uma simulação de quebra de amêndoas fermentadas e secas (tomando como base de cálculo 10000g) utilizando as informações da Tabela 11 e do fluxograma da Figura 1, tendo como finalidade ter-se uma idéia do percentual de pureza do material final obtido. As velocidades e ensaio escolhidos estão demonstrados na Tabela 12. As velocidades dos blocos foram escolhidas ao acaso, com exceção do bloco 2 (explicado anteriormente) e do bloco 1, pois não é possivel escolher outros ensaios nos bloco 1 sem ser o A ou B, pelo fato que os estudos que foram feitos nos blocos seguintes $(2,3$, e 4$)$ foram com materiais que apresentavam umidade de 5\% na amêndoa inteira.

TABELA 11. Tabela de distribuição das massas no fluxograma da Figura 1 durante os ensaios de quebra e separação.

\begin{tabular}{|c|c|c|c|c|c|}
\hline \multirow{3}{*}{ Blocos } & \multirow{3}{*}{ Ensaios } & \multirow{2}{*}{\multicolumn{2}{|c|}{$\begin{array}{l}\text { \% do material, após a } \\
\text { quebra, que ficou retido } \\
\text { nas peneiras para as } \\
\text { próximas etapas de } \\
\text { quebras e separação. }\end{array}$}} & \multirow{3}{*}{$\begin{array}{c}\text { \% de nibs obtido } \\
\text { (material que } \\
\text { passou pela } \\
\text { peneira de } 2 \mathrm{~mm} \text { ). }\end{array}$} & \multirow{3}{*}{$\begin{array}{c}\% \text { de cascas nos } \\
\text { nibs obtidos }\end{array}$} \\
\hline & & & & & \\
\hline & & $4,76 \mathrm{~mm}$ & $2 \mathrm{~mm}$ & & \\
\hline \multirow{10}{*}{ Bloco 1} & A & 52,86 & 22,24 & 23,99 & 0,4030 \\
\hline & $\mathrm{B}$ & 47,76 & 23,64 & 26,95 & 0,3459 \\
\hline & C & 78,24 & 12,4 & 8,833 & 0,6026 \\
\hline & $\mathrm{D}$ & 71,15 & 17,06 & 11,11 & 0,3447 \\
\hline & E & 59,23 & 20,31 & 19,59 & 0,3756 \\
\hline & $\mathrm{F}$ & 55,51 & 22,06 & 21,76 & 0,2385 \\
\hline & G & 58,65 & 21,42 & 19,20 & 0,3532 \\
\hline & $\mathrm{H}$ & 57,17 & 22,43 & 20,25 & 0,2960 \\
\hline & Velocidades & & & & \\
\hline & $\begin{array}{l}\text { Diferenciais } \\
\quad(\mathrm{m} / \mathrm{s})\end{array}$ & $3,35 \mathrm{~mm}$ & $2 \mathrm{~mm}$ & & \\
\hline \multirow{3}{*}{ Bloco 3} & 0,84 & $43,31 \pm 2,88$ & $25,21 \pm 7,99$ & $34,49 \pm 0,65$ & $1,360 \pm 0,25$ \\
\hline & 1,87 & $44,83 \pm 4,66$ & $20,81 \pm 2,25$ & $34,25 \pm 3,045$ & $1,656 \pm 0,46$ \\
\hline & 3,23 & $40,75 \pm 2,34$ & $21,47 \pm 0,56$ & $36,85 \pm 1,76$ & $2,212 \pm 0,38$ \\
\hline \multicolumn{6}{|c|}{$2 \mathrm{~mm}$} \\
\hline \multirow{3}{*}{ Bloco 4 a } & 0,84 & 36,73 & $3 \pm 1,63$ & $62,31 \pm 2,49$ & $1,402 \pm 0,46$ \\
\hline & 1,87 & 34,23 & $3 \pm 2,51$ & $64,99 \pm 1,70$ & $1,024 \pm 0,28$ \\
\hline & 3,23 & 32,07 & $\pm 0,92$ & $67,37 \pm 1,50$ & $0,948 \pm 0,17$ \\
\hline \multirow{3}{*}{$\begin{array}{l}\text { Bloco } 4 \text { b } \\
\left(2^{\circ} \text { vez }\right)\end{array}$} & 0,84 & 63,82 & $2 \pm 1,43$ & $36,47 \pm 1,50$ & $2,660 \pm 0,77$ \\
\hline & 1,87 & 64,60 & $\pm 1,13$ & $35,83 \pm 3,28$ & $2,386 \pm 0,26$ \\
\hline & 3,23 & 64,10 & $\pm 2,44$ & $35,04 \pm 4,61$ & $2,378 \pm 0,38$ \\
\hline
\end{tabular}

TABELA 12. Ensaio e velocidades escolhidas em cada bloco para a simulação de quebra de $10 \mathrm{~kg}$ de amêndoas de cupuaçu fermentadas e secas.

\begin{tabular}{cccccc}
\hline & Bloco1 & Bloco2 & Bloco 3 & Bloco 4a & Bloco 4b \\
\hline $\begin{array}{c}\text { Ensaio } \\
\text { Velocidades }\end{array}$ & A & 3,23 & 3,23 & 0,84 & 0,84 \\
$\begin{array}{c}\text { Diferenciais } \\
(\mathrm{m} / \mathrm{s})\end{array}$ & & & & & \\
& & & & & \\
\end{tabular}

Para melhor visualização, toda esta distribuição de massa está demonstrada através do fluxograma da Figura 2.

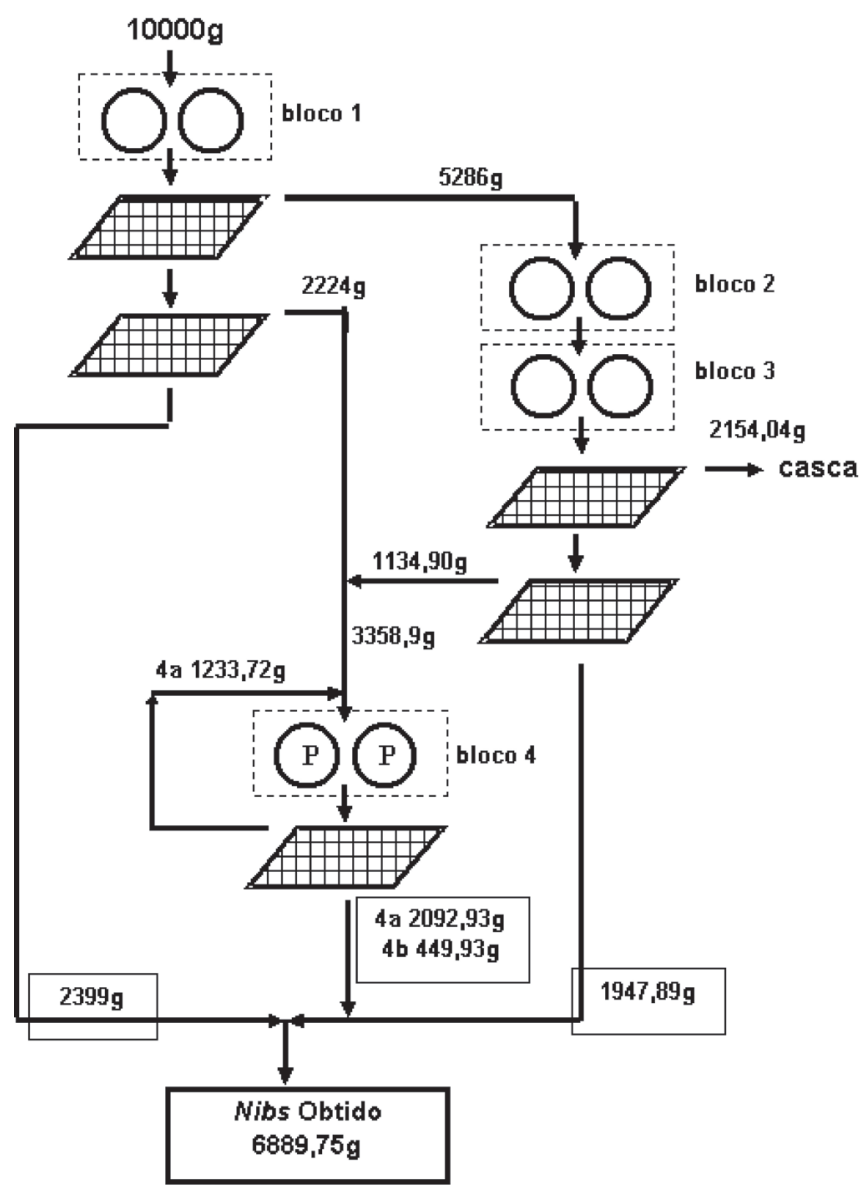

FIGURA 2. Visualização do direcionamento das massas em uma quebra de amêndoas simulada com ensaio e velocidades determinados.

Pela simulação de quebra, o bloco 1 forneceu $2399 \mathrm{~g}$ de nibs e o bloco 3 forneceu 1947,89g. O bloco 4 na primeira passagem (4a) forneceu 2092,93g de nibs e, na segunda passagem (4b), 449,93g de nibs. A massa de material total obtido no fluxograma foi de $6889,75 \mathrm{~g}$ de nibs, pronta para ser processada para a confecção do cupulate $^{\circledast}$. Também através da Tabela 11 , sabe-se qual é o percentual de casca contida nos nibs fornecidos em cada bloco. Agora é necessário fazer um balanço de massa do percentual de casca para descobrir a pureza do material final obtido.

O valor da massa de casca é fornecido pela seguinte fómula:

$$
\mathrm{M}_{\text {casca }}=\left(\mathrm{M}_{\text {cotil. }} \mathrm{xPC}\right) / 100
$$

Sendo:

$\mathrm{M}_{\text {casca }}=$ Massa de casca (g).

$\mathrm{M}_{\text {cotil. }}=$ Massa de nibs (g).

$\mathrm{PC}=$ Percentual de casca contida nos nibs (\%).

O balanço de massa de casca nos nibs é fornecido pela fórmula abaixo:

$M_{\text {cascafinal }}=\sum M$ cascaentra 
Sendo:

$\mathrm{M}_{\text {cascafinal }}=$ Massa de casca contida nos nibs final do fluxograma de quebra (g).

$\mathrm{M}_{\text {cascaentra }}=$ Massa de casca fornecida por um determinado bloco (g).

Utilizando a fórmula do balanço de massa de casca, juntamente com os valores das massas dos nibs fornecidos por cada bloco na simulação de quebra, com seus respectivos percentuais de casca (Tabela 11), obtem-se o valor do percentual de casca no material final.

\begin{tabular}{|c|c|c|c|c|}
\hline & Bloco 1 & Bloco 3 & Bloco $4 a$ & Bloco $4 b$ \\
\hline $6889,75 x P$ & $2399 \times 0,4030$ & $1947,89 \times 2,212$ & $2092,93 \times 1,402$ & $449,93 \times 2,660$ \\
\hline 100 & 100 & 100 & 100 & 100 \\
\hline
\end{tabular}

$$
\mathrm{P}=1,36 \%
$$

O percentual de casca da matéria-prima total obtida apresenta um valor muito bom, sendo que o roteiro utilizado para as etapas de quebras destas amêndoas no fluxograma foi o que apresentava as maiores quantidades de cascas e gérmen. Em termos de rendimento, o percentual de cotilédone em uma massa de amêndoa de cupuaçu gira em torno de $72,44 \%$ (Tabela 2 , média de F1 com F3), e as 10000g de amêndoas fragmentadas na simulação apresentavam em torno de $7244 \mathrm{~g}$ de cotilédone. Como foram obtidos 6889,75g de nibs, o rendimento de quebra foi de $95,10 \%$.

\section{4 - CONCLUSÕES}

O sistema proposto de quebra de amêndoas através de um beneficiador de cilindros, seguido de separação das frações através do método de peneiramento, mostrou-se uma forma alternativa mecanizada válida, com bons resultados tanto na parte de rendimento na obtenção dos nibs, quanto nos valores de pureza deste material.

A umidade de $5 \%$ da amêndoa inteira mostrou a maior influência na quebra das amêndoas junto com os melhores resultados na obtenção do material final, e quanto menor o valor da umidade, maior o percentual dos nibs obtidos. A velocidade diferencial entre os cilindros não apresentou diferença significativa na obtenção da quantidade dos nibs (com exceção do bloco 4a), mas no caso do bloco 1 , se for incluído o parâmetro velocidade diferencial no modelo matemático, haverá diminuição dos erros entre valores preditos e observados.

O material final obtido após todas as etapas de quebra e peneiramento, mostrou percentuais de casca e gérmen inferiores a $1,75 \%$. A metodologia também apresenta bom rendimento total de nibs obtidos, e valores iguais ou superiores a $90 \%$ de cotilédone recuperado.

\section{5 - REFERÊNCIAS BIBLIOGRÁFICAS}

[1] AOAC. Official Methods of Analysis of the Association of Official Analytical Chemists. Washington: edited Ig W. Horwitz $16^{a}$ ed., 1997. v. 2, Cap. 31, p. 1.
[2] AYDIN, C. Physical properties of hazel nuts. Biosystems Engineering, v. 82, n. 3, p. 297-303, 2002.

[3] BARROS NETO, B.; SCARMINIO, I.S.; BRUNS, R.E. Como fazer experimentos: pesquisa e desenvolvimento na ciência e na indústria. Campinas: Editora da Unicamp, 2001. 401p.

[4] CODEX ALIMENTARIUS COMMISSION, 24, 2001, Geneva. Distribution of the report of the eighteenth session of the Codex Committee on Chocolate and Chocolate Products (ALINORM 01/04). Rome: 2001. $49 \mathrm{p}$.

[5] LOMAURO, C.J.; BAKSHI, A.S.; LABUZA, T.P. Evaluation of food moisture sorption isotherm equations. Part I: fruit, vegetable and meat products. Lebensmittel Wissenschaft und Technologies, v. 18, p. 111-117, 1985.

[6] LOPES, A.S. Estudo químico e nutricional de amêndoas de cacau (Theobroma cacao L.) e cupuaçu (Theobroma grandiflorum Schum) em função do processamento. Campinas, 2000. 112p. Dissertação (Mestre em Tecnologia de Alimentos) - Faculdade de Engenharia de Alimentos, Universidade Estadual de Campinas (UNICAMP).

[7] MATTIETTO, R.A. Estudo comparativo das transformações estruturais e físico-químicas durante o processo fermentativo de amêndoas de cacau (Theobroma cacao L.) e cupuaçu (Theobroma grandiflorum Schum). Campinas, 2001. 164p. Dissertação (Mestre em Tecnologia de Alimentos) - Faculdade de Engenharia de Alimentos, Universidade Estadual de Campinas (UNICAMP).

[8] MINIFIE, B.W. Chocolate, cocoa and confectionery: science and technology. 3. Ed. New York: Van Nostrand Reinhold, 1989. p. 47-52.

[9] NAZARÉ, R.F.R. Processamento tecnológico do cupuaçu (Theobroma grandiflorum Schum). I WORKSHOP SOBRE AS CULTURAS DE CUPUAÇU E PUPUNHA. 1996, Manaus. Anais. Manaus - AM: Embrapa - CPAA, 1996. p.136-142.

[10] QUEIROZ, M.B. Estudo dos parâmetros de torração de amêndoas de cupuaçu (Theobroma grandiflorum). Campinas, 1999. 109p. Dissertação (Mestrado) - Faculdade de Engenharia de Alimentos, Universidade Estadual de Campinas (UNICAMP).

[11] VASCONCELOS, M.A.M. de. Transformações físicas e químicas durante a fermentação de amêndoas de cupuaçu (Theobroma grandiflorum Schum). Campinas, 1999. 114p. Dissertação (Mestrado) - Faculdade de Engenharia de Alimentos, Universidade Estadual de Campinas (UNICAMP).

\section{6 - AGRADECIMENTOS}

Os autores expressam seus agradecimentos ao CNPq pela Bolsa de Mestrado concedida à associada ao presente trabalho.

Nota: A metodologia de quebra e separação para obtenção dos nibs das amêndoas de cupuaçu, apresentado neste trabalho, foi protocolizado no INSTITUTO NACIONAL DA PROPRIEDADE INDUSTRIAL (INPI) com o número PI0304.042-9 (PATENTE REQUERIDA). 